\title{
MÔ PHÓNG MộT HỆ ĐộNG HỌC PHI TUYẾN, KHÔNG DỪNG CHỊU TÁC ĐộNG CỦA NHIỄU
}

\author{
PHAN VĂN TƯ
}

\begin{abstract}
This paper is using SIMULNK for the model building of the dynamic nonlinear, time-variable system under acting of a noise, e.g. the control system of fly equipment. These results showed the noise influence on system variables, that gave us decision to use or to resist the noise influence for system control.
\end{abstract}

\section{ĐẶT VẤN Đ产}

Trong thực tế hầu hết các hệ thống điều khiển tự động đều được mô tả toán học bằng các phương trình vi phân phi tuyến, không dưng. Các tác động, trên thực tế, đối với hệ thống là rất phức tạp, nhiều khi là nhiễu ngẫu nhiên.

Để giải các phương trình vi phân đó, hay làm bài toán phân tích các hệ thống động học phức tạp đó người ta đã dùng các phương pháp tuyến tính hóa Ơ-le, Runge-Kutta, Adam... Các phương pháp đó cho ta lời giải là các điểm ứng với các thông số được chọn cố định (đông cứng).

Hiện nay đã xuất hiện nhiều công cụ mô phỏng trong đó có Matlab-Simulink $[1,2]$. Với công cụ này ta có thể xây dựng mô hình tương ứng với hệ thống như nó vốn có và khảo sát các quá trình xảy ra trong đó. Qua bài báo này chúng ta có thể đánh giá được sức mạnh và tính ưu việt của phương pháp mới và cố gẳng đưa nó vào giải quyết những vấn đề gặp phải của chính mình.

\section{PHÂN TÍCH BÀI TOÁN}

Một hệ động học phức tạp được mô tá bằng hệ phương trình vi phân sau đây:

$$
\left\{\begin{array}{l}
\dot{r}=V \cos (\theta-\varepsilon) \\
\dot{\varepsilon}=\frac{1}{r} \sin (\theta-\varepsilon) \\
\dot{\theta}=\frac{w}{v} \\
\dot{w}=-\frac{1}{T} w+\xi(t) \\
v=0
\end{array}\right.
$$

với các điều kiện đầu

$$
r(0)=100.000 \mathrm{~m}, \varepsilon(0)=0,2 \mathrm{rad}, \theta(0)=3,14 \mathrm{rad}, v=500 \mathrm{~m} / \mathrm{s}, T=0,005 .
$$

Hệ phương trình này có thể mô tả chuyển động của vật thể bay trong trường hấp dẫn dưới tác động của nhiếu ngẫu nhiên. 
Ơ đây $r$ cự ly đến một điểm mốc nào đó

$v$ - tốc độ, xem như không đổi,

$\theta$ - góc nghiêng của quỹ đạo,

$\varepsilon-$ góc tầm,

$w$ - gia tốc pháp tuyến,

$\xi(t)$ - nhiễu ngẫu nhiên,

$T$ - hằng số thời gian.

Nhìn vào hệ phương trình ta thấy: quan hệ giữa các biến là phi tuyến và thông qua các hàm lượng giác. Cự ly là một biến - nghĩa là luôn luôn thay đổi làm cho hệ phương trình trở thành không dừng. Hệ lại chịu tác động của nhiễu ngẫu nhiên $\xi(t)$.

Đây là một mô hình toán đã được đơn giản hóa nhiều so với thực tế nhưng chúng ta vẫn thấy nó khá phức tạp. Để giải quyết bằng công cụ lập trình trước đây sẽ gặp phải rất nhiều khó khăn và độ chính xác sẽ bị hạn chế.

\section{THU ẬT TOÅN GIẢI}

Xuất phát từ hệ phương trình mô tá động học ta có thể xây dựng sơ đồ thuật toán mô phỏng bằng các khối như:

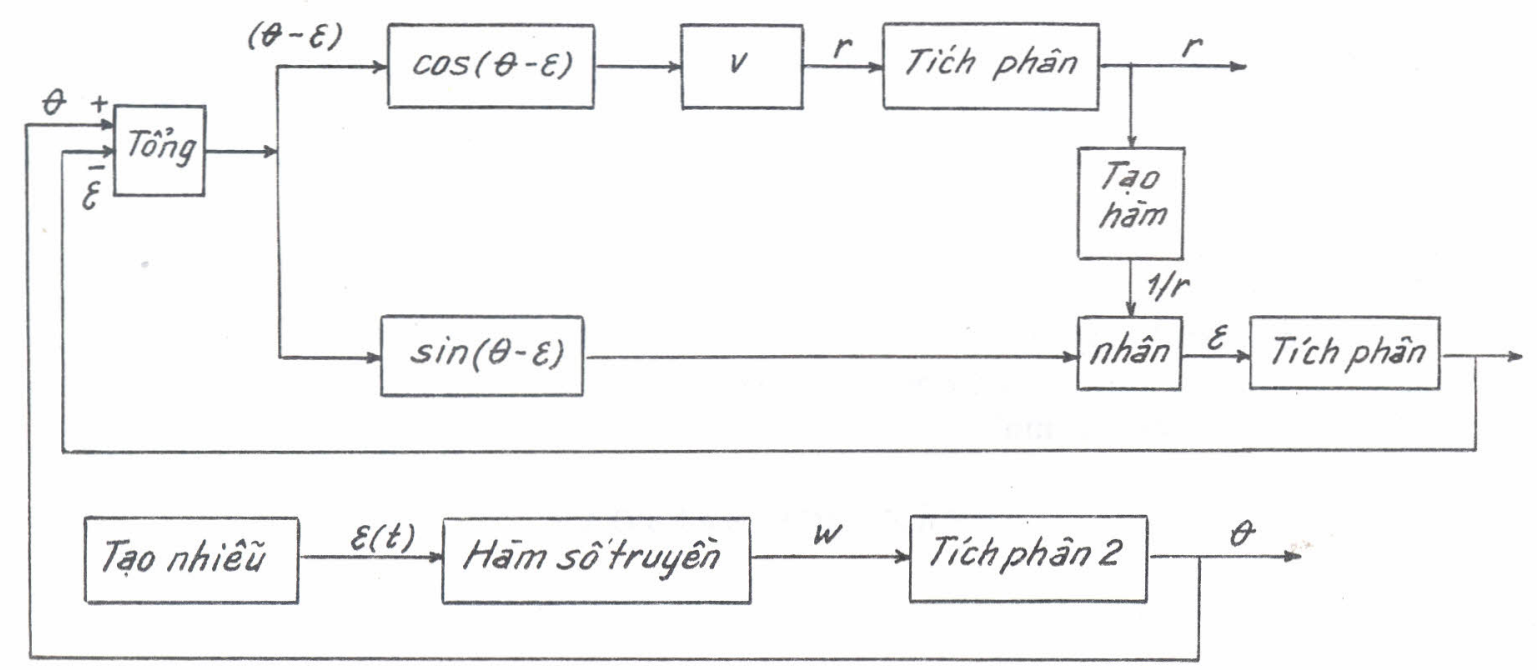

Hinh 1

\section{SƠ ĐỒ CẤU TRÚC THEO SIMULINK}

Simulink [2] là từ viết tắt của các từ simulation link, nghĩa là liên kết mô phỏng. Nó là một phần mềm có thư viện chứa sẵn các khối động học đã được xây dựng, cho phép ta liên kết các khối đó theo cẩu trúc động học của hệ thống và quan sát kểt quá mô phỏng dưới dạng các đường cong như trên Scop. Khi ta vào Simulink ta có thể lấy các khối động học có sẵn trong thư viện của nó. Ngoài ra ta có thể dùng bộ tạo hàm và dùng công cự tạo hàm của Matlab để tạo ra các hàm cần thiết. Trên sơ đồ ta dùng các Scop để quan sát kết quả (hình 2). 


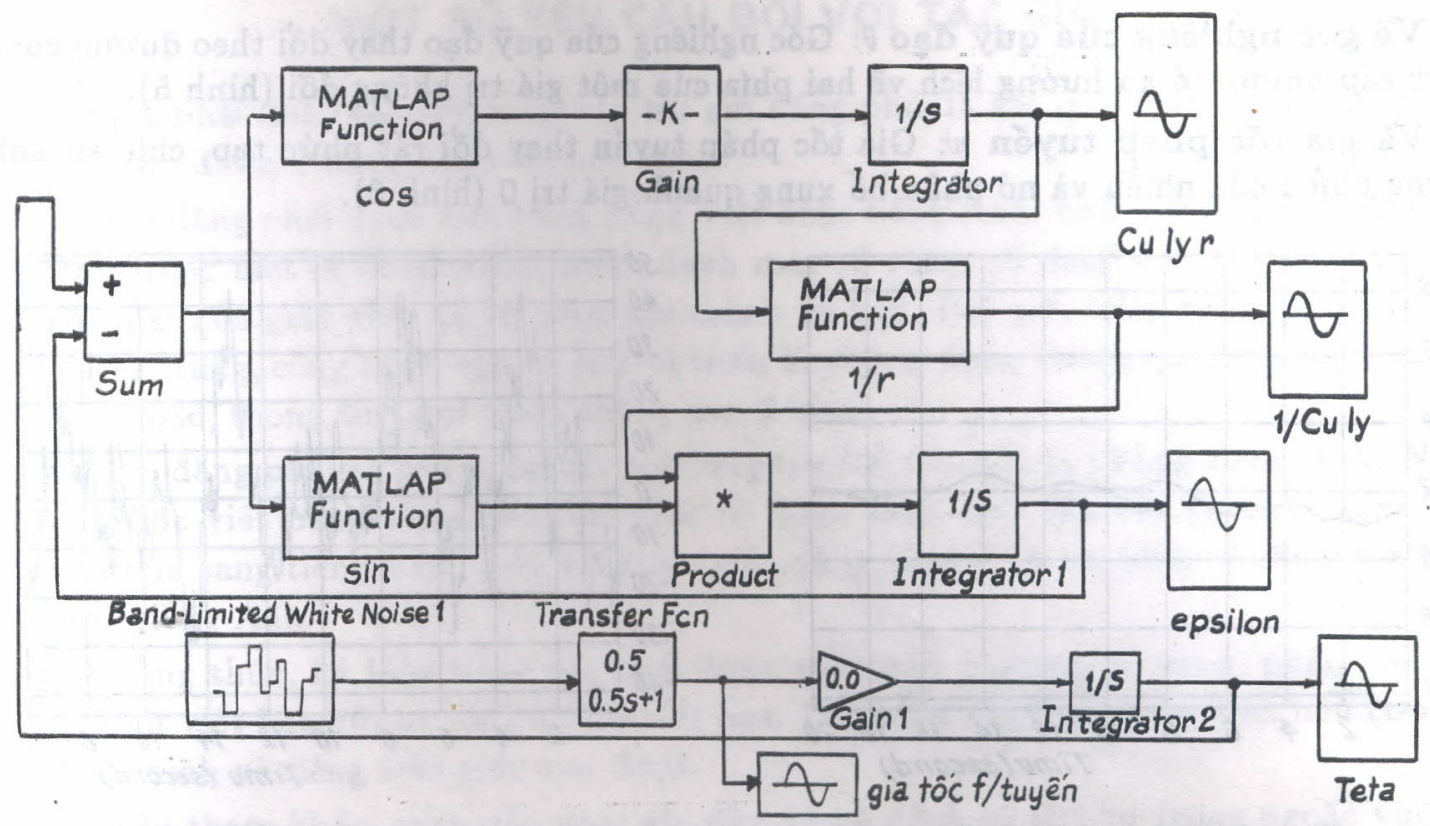

Hinh 2. Sơ đồ cấu trúc theo Simulink

\section{PHÂN TÍCH KÊTT QUẢ VÀ KÊTT LUẬN}

Với công cụ mô phóng Simulink ta đã giải quyết bài toán phức tạp trên bằng thuật toán cấu trúc có tính trực quan cao. Kết quá được ghi nhận cho ta sự hiểu biết về quá trình thay đổi các thông số cưa hệ thống.

Về cự ly $r$ : Ta thấy cự ly giám dần tuyến tính và không chịu ánh hướng của nhiêxu. Nếu như đối tượng mô tá là mục tiêu thì nó càng ngày càng tiến gần đến vị trí quan sát (hình 3).

Về góc tầm $\varepsilon$ : Trong thòi gian quan sát góc tầm hầu như không thay đổi (hình 4 ).

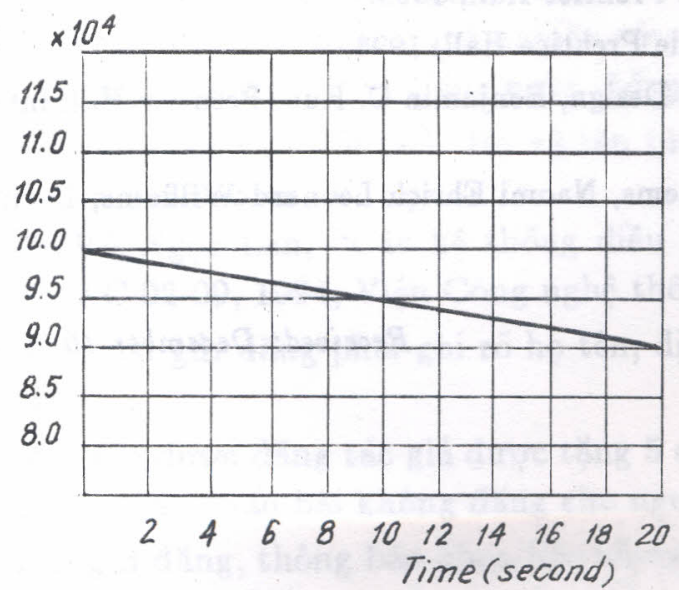

Hinh 3. $r$ thay đổi cự $\mathrm{ly}(\mathrm{m})$

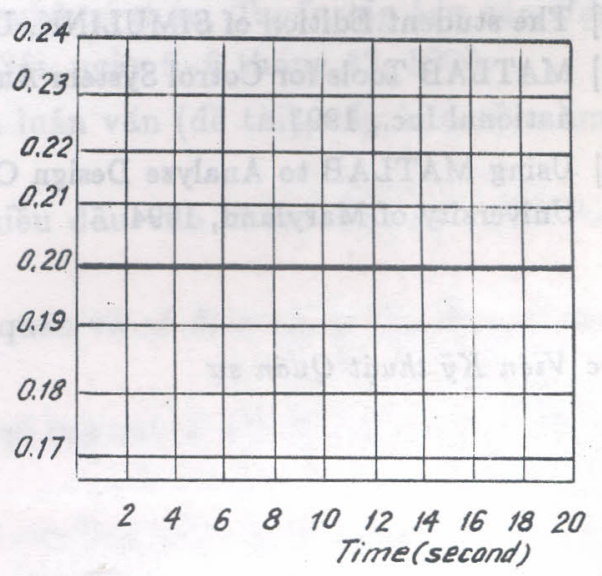

Hinh 4. $\varepsilon$ góc tầm (rad) 
Về góc nghiêng của quỹ đạa $\theta$ : Góc nghiêng của quỹ đạo thay đổi theo đường cong phức tạp nhưng có xu hướng lệch về hai phía cưa một giá trị không đổi (hình 5).

Vè̀ gia tốc pháp tuyển $w$ : Gia tốc pháp tuyến thay đối rất phức tạp, chịu sự ánh hường nhiều của nhiễu và nó phân bố xung quanh giá trị 0 (hình 6).

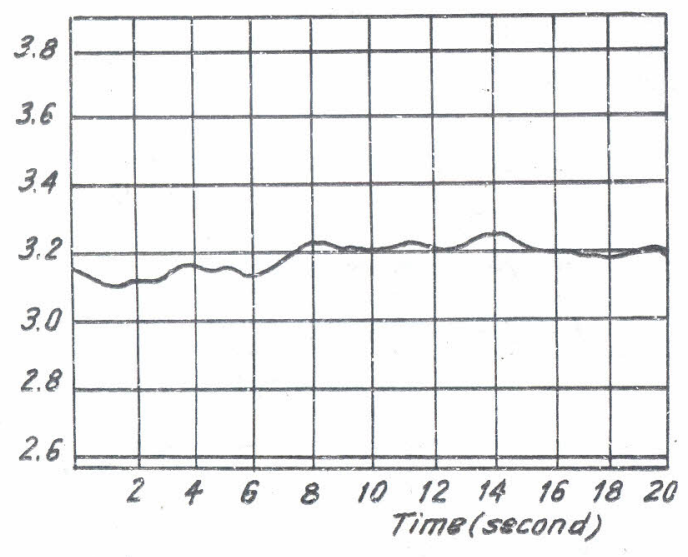

Hìn 5. $\theta$ góc nghiêng của quỹ đạo

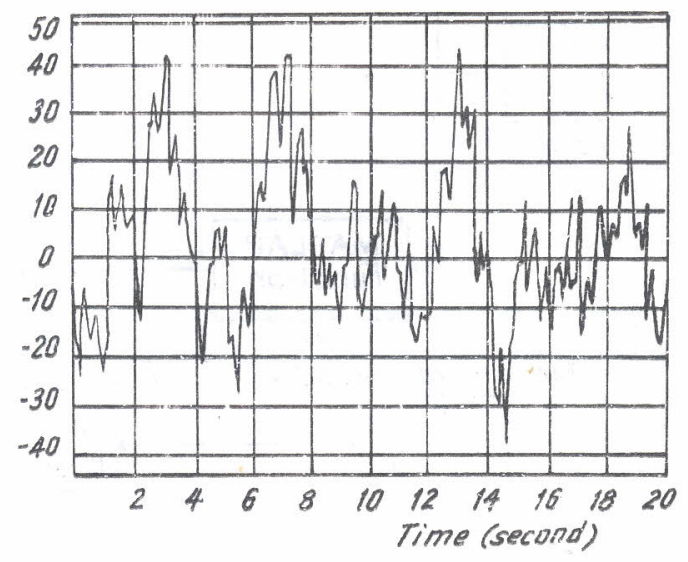

Hinh 6. $w$ gia tốc pháp tuyển $\left(\mathrm{m} / \mathrm{sec}^{2}\right)$

Với kết quá trên ta đưa ra quyết định như th ees nào?

a) Nểu thiết bị bay là của ta thì ta yên tâm rằng quỹ đạo bay vẫn bình thường và ít chịu sự tác động cưa nhiễu ngẫu nhiên.

b) Nếu thiết bị bay cưa đối phương và nó là mục tiêu phái chống trá của ta mà ta muốn gây nhiễu cho nó thì ta phải tăng mạnh cường độ nhiếu mới làm cho nó chệch quỹ đạo.

Công cụ mô phóng đã cho ta kết quá rất gần với thực tế và rất hữu ích.

\section{TÀI LIỆU THAM KHÁO}

(1) The student Edition of MATLAB, User's Guide Prentice Hall, 1993.

[2] The student Edition of SIMULINK, User's Guide Prentice Hall, 1993.

[3] MATLAB Tools for Cotrol System Analysis and Design, Benjamin C. Kuo, Prentice Hall International Inc., 1993.

[4] Using MATLAB to Analyze Design Cotrol Systems, Naomi Ehrich Leonard Williams, Levine, University of Maryland, 1994.

Received: December 10, 1997

Học Viện Kũ thuật Quân sự 